\title{
Comparative Pharmacognostical, Phytochemical and Biological Evaluation of Five Ocimum Species
}

\author{
Sharada L Deore*, Shital R. Ingole, Bhushan A Baviskar, Anjali A. kide
}

Sharada L Deore*, Shital R. Ingole, Bhushan A Baviskar, Anjali A. kide

Govt. College of Pharmacy, Amravati-444 604, Maharashtra, INDIA.

\section{Correspondence}

\section{Sharada L Deore}

Govt. College of Pharmacy, Amravati-444 604, Maharashtra, INDIA

E-mail: sharudeore_2@yahoo.com

History

- Submission Date: 07-09-2020;

- Review completed: 03-11-2020;

- Accepted Date: 10-11-2020.

DOI : 10.5530/pj.2021.13.59

Article Available online http://www.phcogj.com/v13/i2

Copyright

(C) 2021 Phcogj.Com. This is an openaccess article distributed under the terms of the Creative Commons Attribution 4.0 International license.

\section{ABSTRACT}

There are about 150 species of Ocimum in the world and out of that 68 species are found in India. Comparative pharmacognostic study of these Ocimum species is unspecified. Growing demand of Ocimum plants demands quality standards for correct identification of desired Ocimum species. Objectives: Hence aim of present study is to establish comparative pharmacognostical, phytochemical and biological standards for most commonly found and morphologically confusing five species of Ocimum. Methods: Macroscopic, microscopic, preliminary phytochemical evaluations, extraction of essential oils, TLC analysis, in vitro antioxidant and antimicrobial potency of selected five species carried out and compared. Results: This comparative study reports that $O$. sanctum should be preferred in medicine use among selected five species based on phytochemical composition, antioxidant and antimicrobial potency.

Key words: Ocimum americanum, Ocimum basilicum, Ocimum kilimandscharicum, Ocimum gratissimum, Ocimum sanctum.

\section{INTRODUCTION}

Medicinal properties of Ocimum species belongs to mint or lamiaceae family are known for thousand years to various civilizations of the world ${ }^{1-4}$ through Ayurveda, Siddha and Unani systems of medicine..$^{5-9}$ In the Indian subcontinent, in Ayurveda and Indian mythology, it is commonly called as Tulsi means "matchless one" or "incomparable one" and considered as a sacred plant representing Holy Hindu Laxmi Goddess. ${ }^{10-12}$

All parts of this plant species are of major medicinal use. It is useful in treatment of cold, bronchitis, stress, and digestive disorder. This also possesses anti-oxidant, antimicrobial and mosquito repellant properties. It is strong immunomodulator and adoptogen as per ayurveda as this healing herb has ability to enhance the energetic resonance between the body and the environment to protect body from opportunistic infections. ${ }^{13-17}$

Major chemical constituents of Ocimum species are mono and sesquiterpenes like eugenol, $\alpha$-pinene, $\beta$-pinene, camphene, sabinene, p-cymene, limonene, linalool, camphor, borneol, terpin4-ol, a-terpineol, methyl chavicol, $\alpha$-cubebene, $\alpha$-copaene, $\beta$-bourbonene $\beta$-cubebene, $\alpha$-elemene, methyl eugenol, $\beta$-caryophyllene, $\beta$-gurjunene, $\alpha$-humulene, germacrene, germacrene, cubebol and $\delta$-cadinene. ${ }^{18-21}$

In India two forms of Ocimum sanctum (synonym O. tenuiflorum) are more common - dark or Shyama (Krishna) Tulsi and light or Rama Tulsi. Ocimum gratissimum is known as Vana (wild/forest) tulsi. Various other species are also commonly found in India like O. canum, O. basilicum, $\mathrm{O}$. kilimandscharicum, O. ammericanum, O. camphora and $O$. micranthum. ${ }^{21-24}$

\section{MATERIALS AND METHODS}

Material collection, identification, authentication

Plant material (Ocimum americanum, Ocimum basilicum) was purchased from local nursery of district Amravati from Maharashtra. Plant material (Ocimum kilimandscharicum, Ocimum gratissimum, Ocimum sanctum) was purchased from Nagarjun Garden Dr. P.D.K.V. Akola. Identification and authentication was made from H.O.D, Botany department, Government Vidarbha Institute of science \& Humanities, Amravati (Maharashtra)

\section{Phamacognostic evaluations}

\section{Macroscopic evaluation}

Macroscopy of whole plant was studied by observing the organoleptic characters such as color, odor, size, shape, taste and special features including touch and texture etc. Organoleptic evaluations can be done by means of organs of special sense which includes the above parameters and thereby define some specific characteristics of the material which can be considered as a first step towards establishment of identity. ${ }^{25-26}$

\section{Microscopic evaluation}

Microscopic evaluation is very helpful in the initial identification of herbs as well as in detection of adulteration by characteristics tissue features. Every plant possesses a characteristics tissue structure which can be demonstrated through study of tissue arrangement, cell walls and configurations, when properly mountain in stains, reagents and media. Thus it determines the size, shape, and relative structure of different cell Microscopical parameters 
observed were, arrangement of tissues in a transverse section, type of epidermal cells, presence and type of calcium crystals, starch grains, oil globules, aleurone grains and trichomes. ${ }^{25}$ Microscopy carried out by using digital microscope (Olympus, Model- U-APT, Cx31RTSF).

\section{Powder microscopy evaluation}

The powder of respective plant was used for powder microscopy study. Plain powder and powder treated with specific reagents like phloroglucinol -hydrochloric acid, iodine solution and Sudan red solution were spread as a thin layer on separate glass sides and observed under microscope. ${ }^{26}$

\section{Physicochemical evaluation}

Ash values, extractive values and loss on drying determined as per standard procedures mentioned in Indian pharmacopoeia $2010 .^{26-}$

27 These physicochemical standards are helpful in comparative determination quality and purity of the powder of plants of Ocimum genus.

\section{Preliminary phytochemical evaluation}

Preliminary phytochemical evaluations is the step to identify different classes of constituent that are primary constituents like carbohydrate, proteins, and lipids or secondary metabolite like glycosides, alkaloids, volatile oil, tannins etc. of great. The compounds that are responsible for medicinal efficacy are usually secondary metabolite. Hence plant material is subjected to preliminary phytochemical screening ${ }^{26}$ for detection of various chemical constituents.

\section{Extraction of essential oil}

Extraction of oil was carried out by hydro distillation technique using Clevenger apparatus ${ }^{26-27}$ and stored in dark glass vial in a refrigerator until further testing.

\section{Chromatographic evaluation}

Comparative TLC using pre-coated silica gel GF254plate as stationary phase, toluene: ethyl acetate (93:7) as mobile phase and Anisaldehydesulphuric acid as spraying reagent. Sprayed plate heated on hot plate at $110^{\circ} \mathrm{C}$ till color developed and intensified. ${ }^{28}$

\section{Antioxidant activity}

Free radical generation due to oxidative stress is one of the major causes of many diseases in human body. Ocimum species are known to be a very good free radical scavenger hence it is decided to know comparative antioxidant potential of selected Ocimum species. DPPH $(1,1$ - diphenyl-2- picryl-hydrazyl) is a stable free radical and methanolic solution of it is used to evaluate the anti oxidant activity of several natural compounds. To $1 \mathrm{ml}$ of DPPH solution, $3 \mathrm{ml}$ of oil sample added. The same reaction mixture without sample but equivalent amount of standard phosphate buffer served as control. Well mixed solution allowed to stand at room temperature for $30 \mathrm{~min}$. Absorbance of reaction mixture was measured at $517 \mathrm{~nm}$. Percentage scavenging activity at different concentrations was calculated by using formula: $\%$ scavenging activity $=1$ - absorbance of test $/$ absorbance of control $\mathrm{x} 100 .{ }^{26}$

\section{Antibacterial activity}

Pathogenic bacteria have always been considered as a major cause of morbidity and mortality in humans. Even though pharmaceutical companies have produced a number of new antibacterial in the last years, antimicrobial resistance has now become a global concern. The global emergence of multi-drug resistant bacteria is increasingly limiting the effectiveness of current drugs and significantly causing treatment failure. In vitro antibacterial potential of essential oils of selected Ocimum species against Escherichia coli was determined by agar well method using nutrient agar media. At the end of incubation, zone of inhibition formed measured in millimeter. Gentamicin is used as standard. . $^{29-30}$

\section{Antifungal activity}

In vitro antifungal potential of essential oils of selected Ocimum species using YEPD agar media against fungi Candida albicans was determined by using agar well method. All steps are same as procedure given in antibacterial activity, only the incubation period for antifungal activity was for 48 hours. At the end of incubation, zone of inhibition formed measured in millimeter. Fluconazole was used as standard. $29-30$

\section{RESULT AND DISCUSSION}

Medicinal use of Ocimum species is abundantly increasing due to its immunomodulator and antioxidant potential. It is commonly used in many marketed or even in homemade herbal tea formulas. There are about 150 species of Ocimum in the world and out of that 68 species are found in India. Comparative pharmacognostic study of these Ocimum species is unspecified. Hence, present research work generates comparative pharmacognostic data of selected five species of ocimum found in Vidarbha region of Maharashtra state.

Total five species of Ocimum i.e. Ocimum sanctum Linn, Ocimum americanum Linn, Ocimum basilicum Linn, Ocimum gratissimum Linn, Ocimum kilimandscharicum guerke are evaluated comparatively for their morphological, microscopical, physiochemical, and phytochemical parameters and also evaluated for their antioxidant and antimicrobial activity by in vitro methods. The whole plants are shade dried and powdered using grinding mill. The powder was stored in airtight container.

Comparative morphological evaluations of all selected five Ocimum species are summarized in Table 1. Microscopy of leaves and stem as well as powder of whole plant of all selected five Ocimum species studied in detail and comparisons are summarised in Table 2 and Table 3.

Microscopical examination of leaf $O$. americanum shows isobilateral lamina covered with cuticle; glandular trichomes with multi-cellular head and multicellular warty covering trichomes. Mid rib with arc shaped vascular bundle consisting of xylem and phloem, three to four layers of collenchymatous tissue present on upper side of vascular bundle whereas stem part shows cork, vascular bundle containing xylem \& phloem, spongy tissue, collenchymatous cells, pith. Powder microscopy shows reticulate xylem vessel, stone cell, epidermal cell, collenchymas, cork cell.

Ocimum basilicum leaf shows the presence of multicelluler curved trichomes, Upper epidermis, collenchymatous cells, upper palsied cell, vascular bundle containing xylem \& phloem, spongy tissue, lower epidermis, stem section shows unicellular covering trichome, epidermal cell, collenchymatous cells, vascular bundle containing xylem \& phloem, spongy tissue, pith, spongy parenchyma. Powder microscopy shows presence of medullary rays lamellar collenchymas, Cork cell, Epidermal cell, Fibers, Stone cell.

Ocimum gratissimum showed Single layered epidermis, multicelluler covering trichomes and glandular trichomes, collenchymatous cells, Vascular bundle consisting of xylem and phloem, Palisade cells and spongy tissue A transverse section of stem showed Shape of section was rectangular Compressed bark cells followed by single layered epidermis Multicelluler covering trichomes and glandular trichomes, Collenchymatous cells, Vascular bundle contains xylem and phloem, Spongy tissue was present at centre. Powder microscopy glandular trichomes with multi-cellular head, Thin-walled fiber with pointed 
Table 1: Comparative morphological evaluated parameters of selected five Ocimum species.

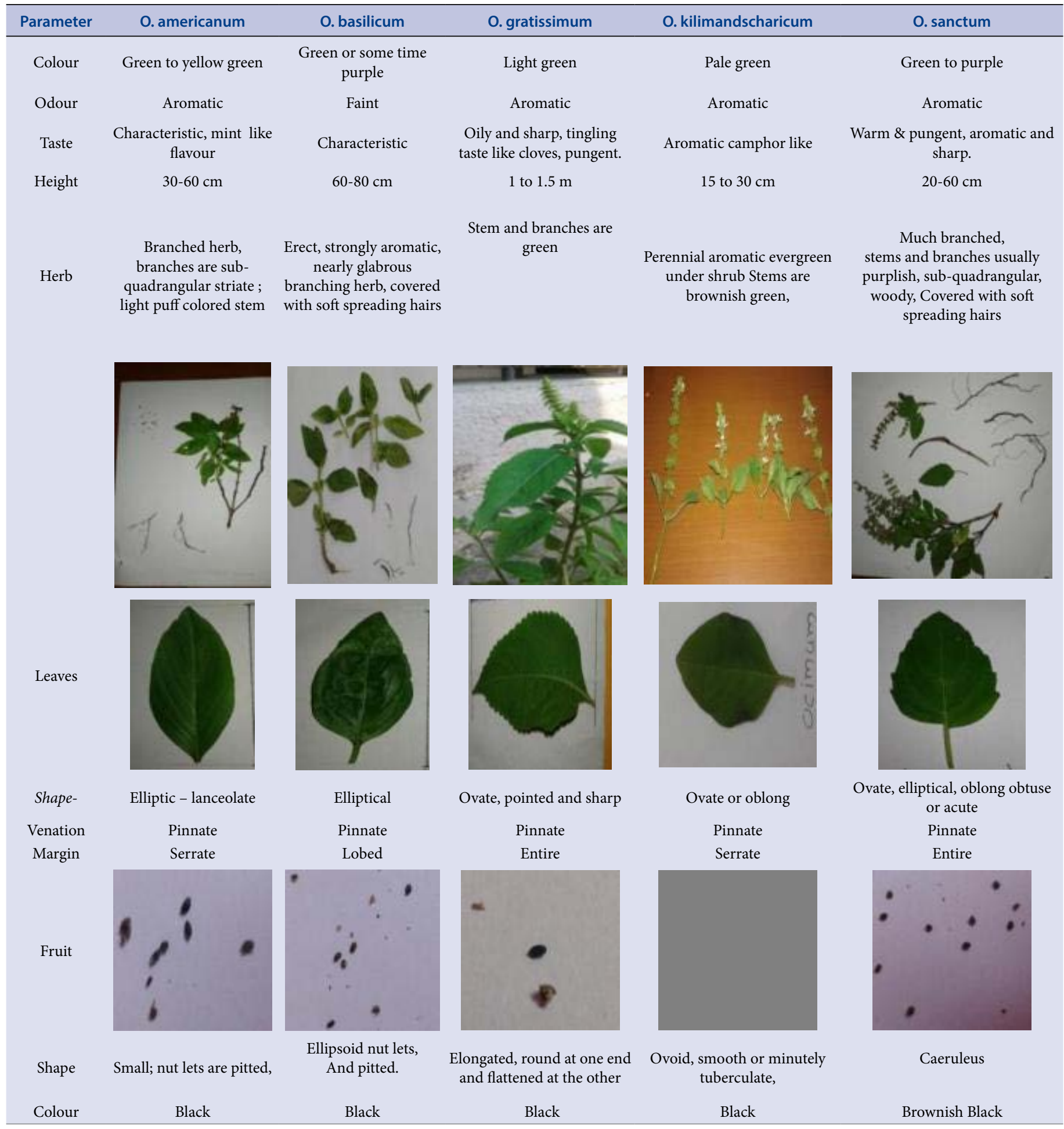




\section{Table 2: Comparative microscopical evaluated parameters of selected five Ocimum species.}
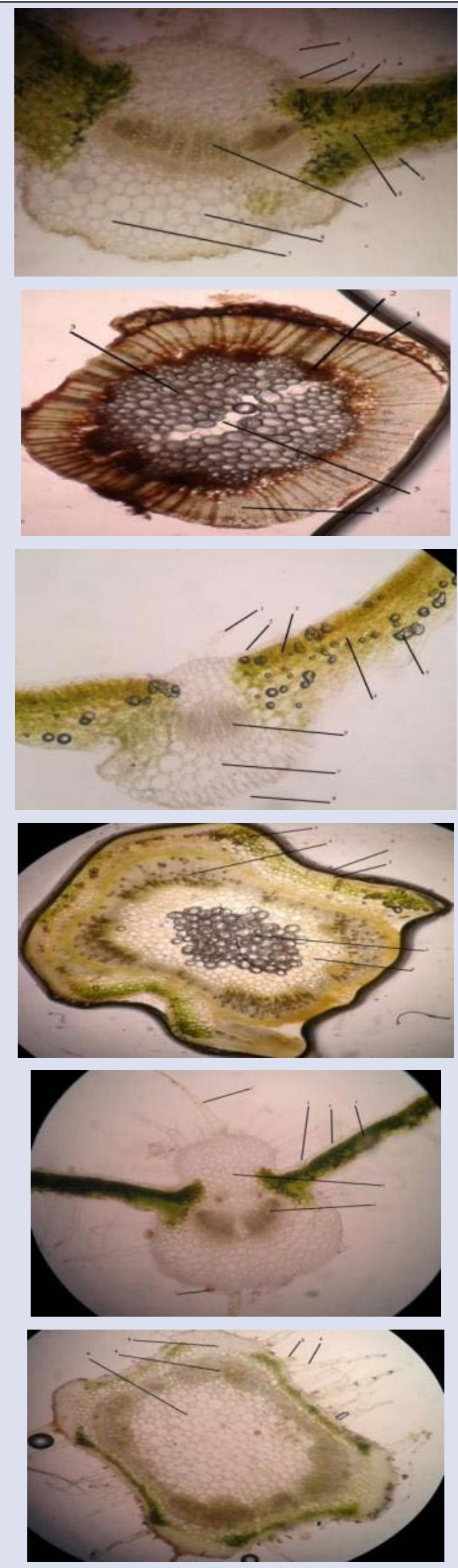

Microscopy leaf of $O$. americanum where 1-Multicellular covering trichomes, 2- Glandular trichomes, 3- Upper epidermis, 4- Upper palisade cell, 5-Vascular bundle containing xylem \& phloem, 6-Collenchymatous cells, 7- Oil globules, 8-Spongy tissue, 9- Lower epidermis

Microscopy of stem of $O$. americanum where1-Cork, 2- Vascular bundle containing xylem \& phloem, 3-Spongy tissue, 4-Collenchymatous cells, 5-pith.

Microscopy leaf of $O$. basilicum where 1-Multicelluler curved trichomes, 2- Upper epidermis, 3-Collenchymatous cells, 4- Upper palsied cell, 5-oil globules, 6-Vascular bundle containing xylem \& phloem, 7- Spongy tissue

8- Lower epidermis

Microscopy stem of $O$. basilicum1-Unicellular covering trichome,

2- Epidermal cell, 3- Collenchymatous cells, 4-Vascular bundle containing xylem \& phloem, 4-Spongy tissue, 5-Pith, 6- spongy parenchyma

microscopy leaf of $O$. gratissimum where 1-Multicellular covering trichomes, 2- Glandular trichomes, 3- Upper epidermis, 4- Upper palsied cell, 5-Collenchymatous cells, 6-Vascular bundle containing xylem \& phloem, 7- oil glands

Microscopy stem of $O$. gratissimum Where 1-Multicellular covering trichome, 2- Glandular trichome, 3-Collenchymatous cells, 4-Vascular bundle containing xylem \& phloem, 5-Spongy tissue 

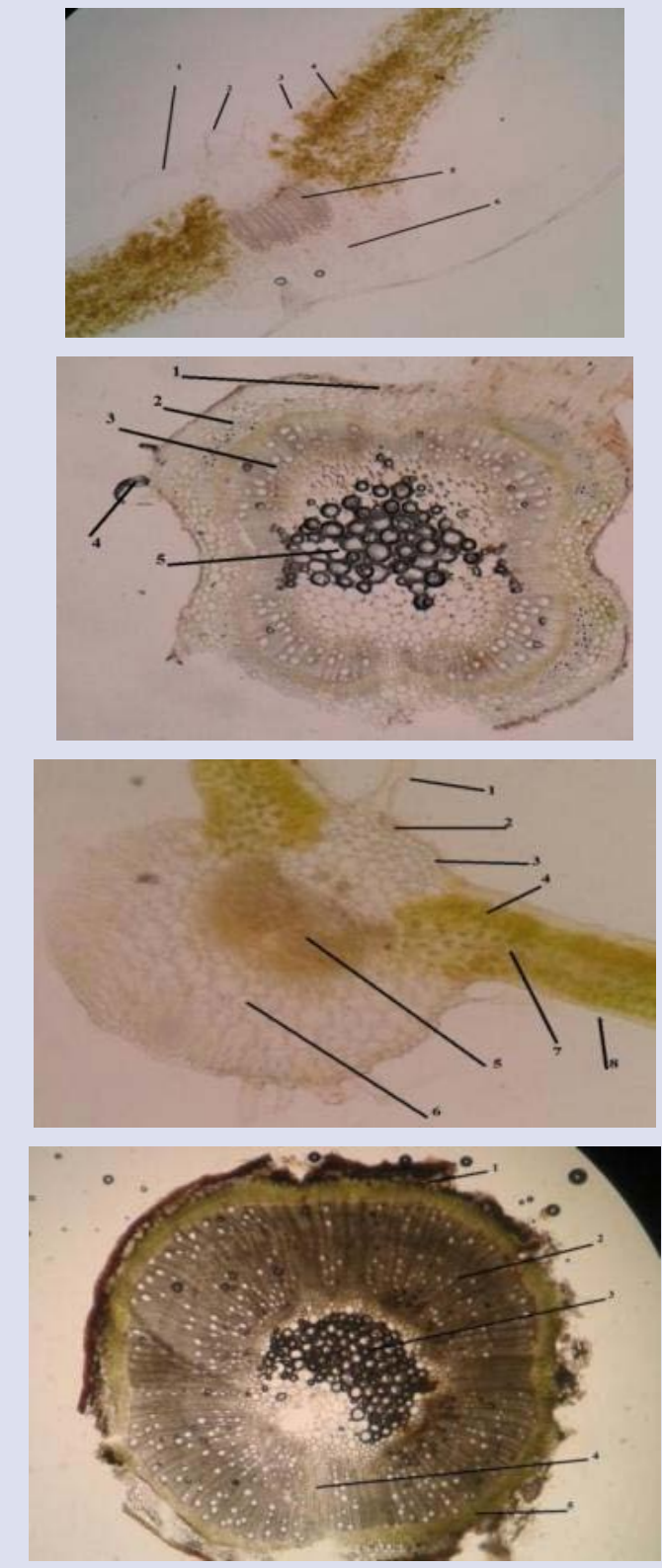

Microscopy of leaf of $O$. kilimandscharicum Where 1-Multicellular covering trichomes, 2Multicelluler curved trichomes, 3- Upper epidermis, 4- Upper palsied cell, 5- Vascular bundle containing xylem \& phloem, 6-Collenchymatous cells

Microscopy of stem of O. kilimandscharicumwhere1-Cork, 2-Collenchymatous cells, 3-Vascular bundle containing xylem \& phloem, 4-Multicelluler curved trichomes, 5-Pith

Microscopy of leaf of o. sanctum where1-Unicellular covering trichomes, 2- Glandular trichomes , 3- Upper epidermis, 4- Upper palsied cell, 5- Vascular bundle containing xylem \& phloem, 6-Collenchymatous cells,7-Parenchyma cell, 8- Epidermal cell

Microscopy stem of O. sanctum 1- Cork, 2- Collenchymatous cells, 3- Pith, 4- Medullar rays

\section{Table 3: Comparative Powder Microscopical evaluated parameters of selected five Ocimum species.}

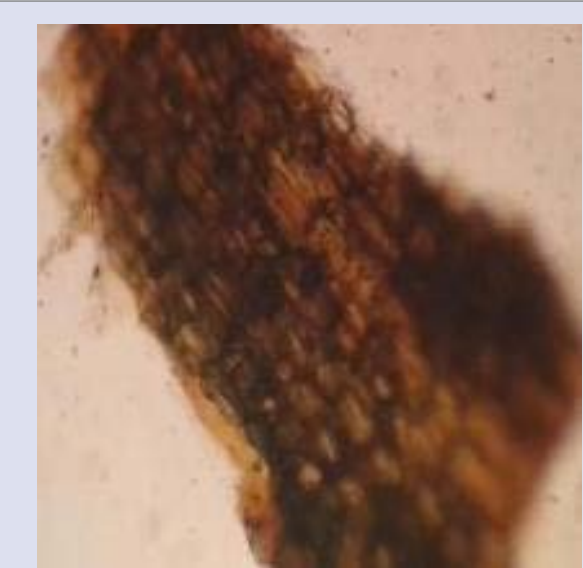

1

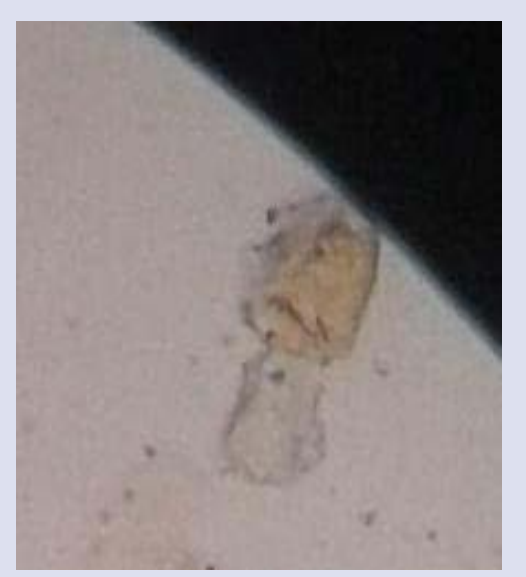

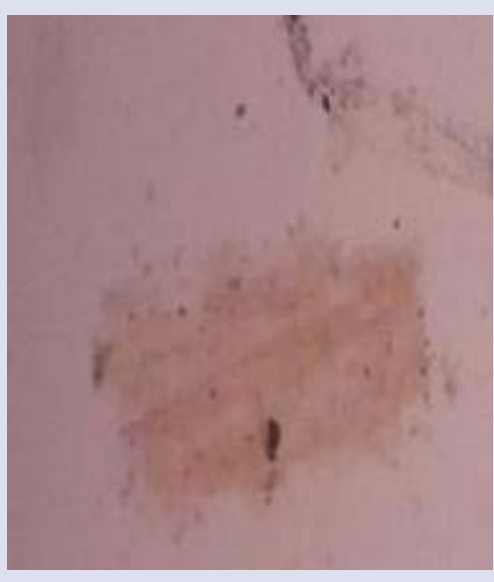




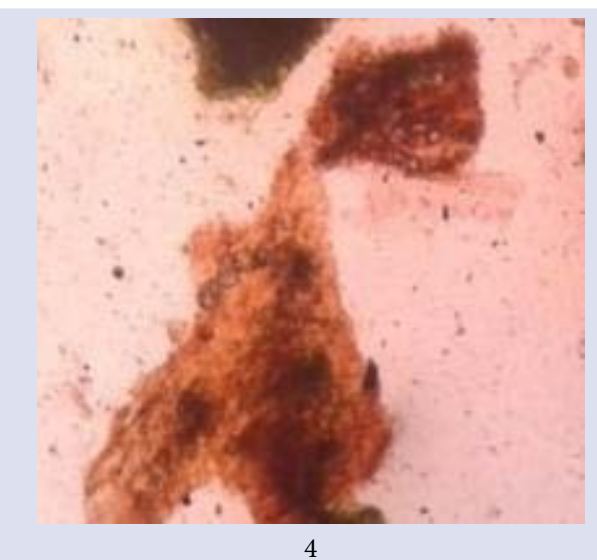

4

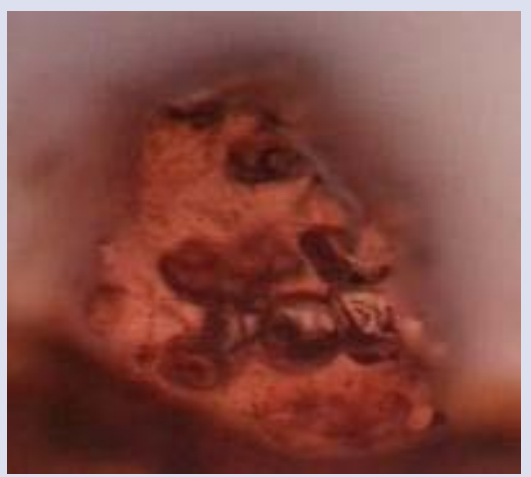

1

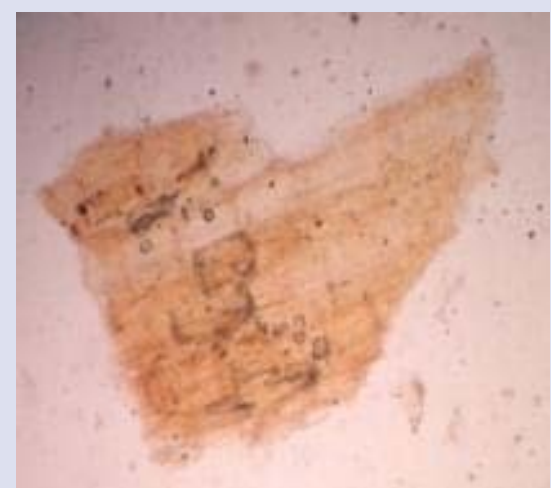

4

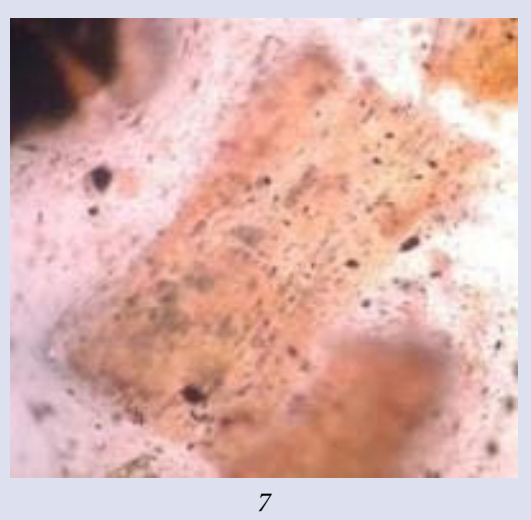

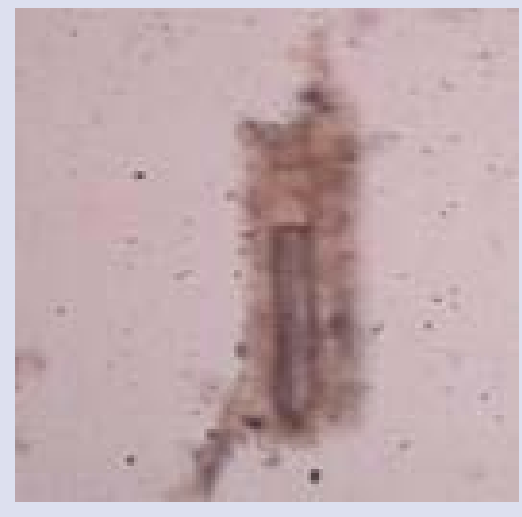

5
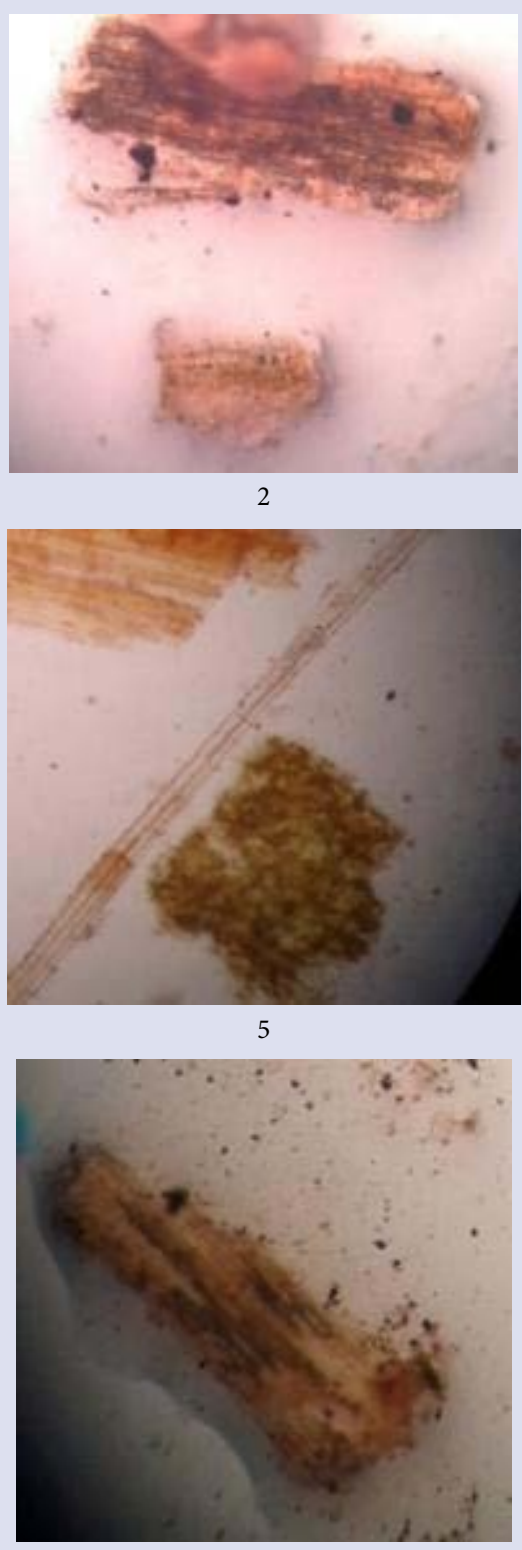

8
Ocimum americanum: 1-Cork cell, 2-Stone cell,

3- epidermal cell, 4-Collenchymas, 5-Reticulate Xylem vessel

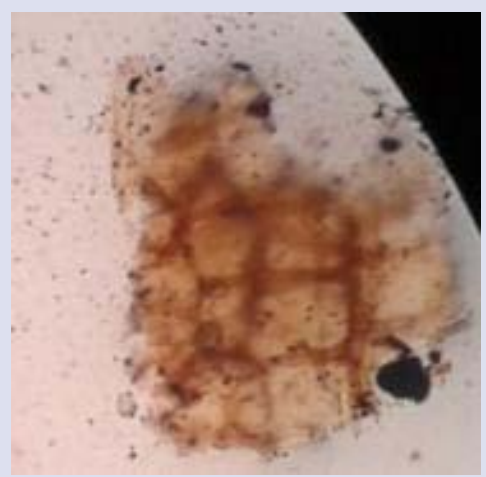

3

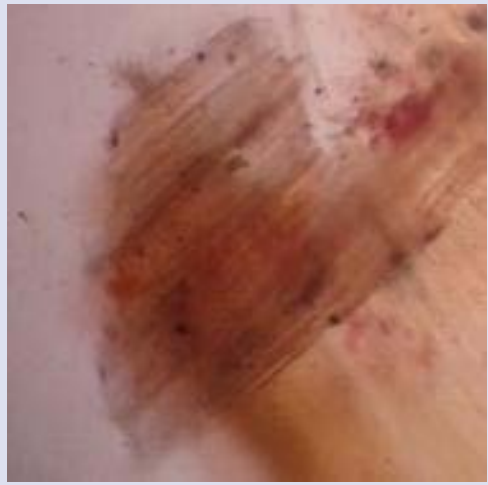

6

Ocimum basilicum : 1-Stone cell, 2-Lamellar collenchymas, 3-Cork cell, 4-Epidermal cell, 5-Fibers, 6-Medullary rays,7-Wood element 


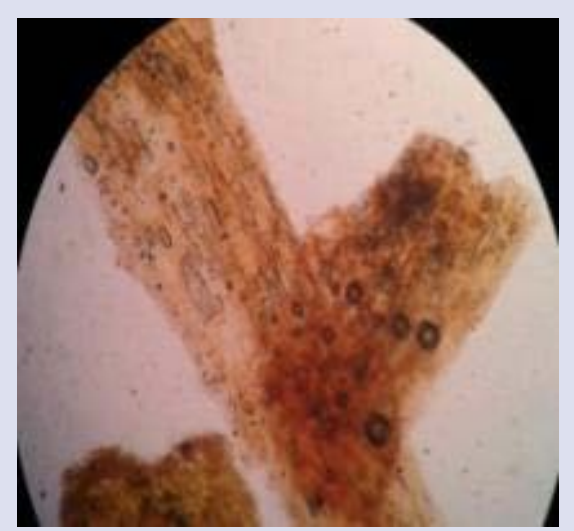

1

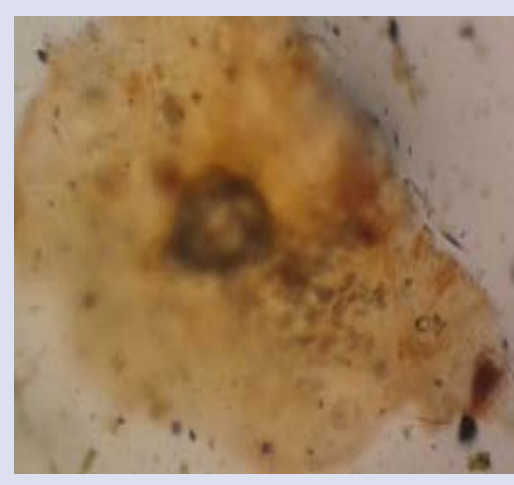

4
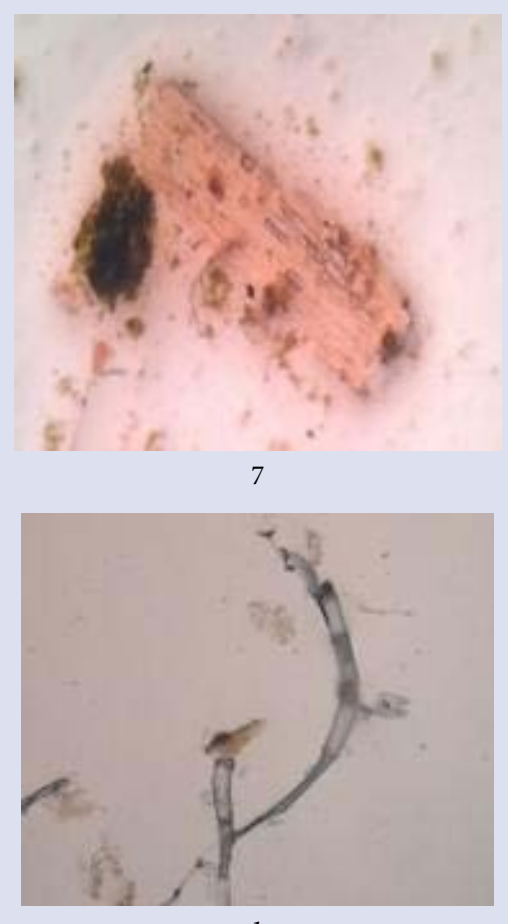

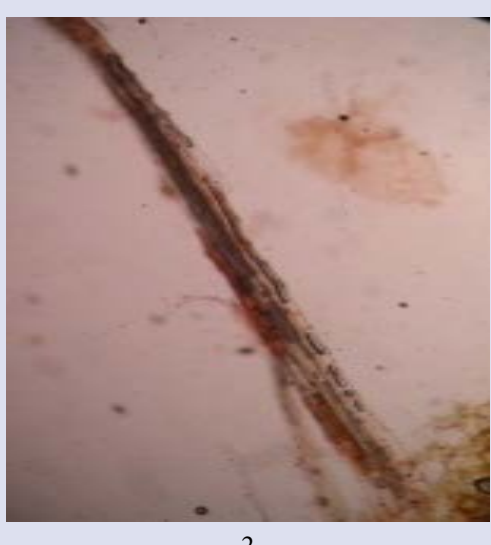

2

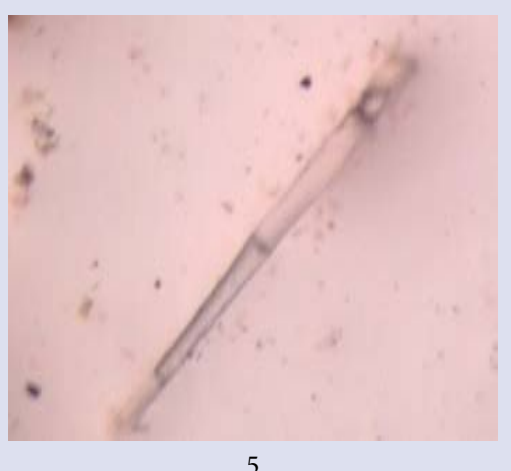

5

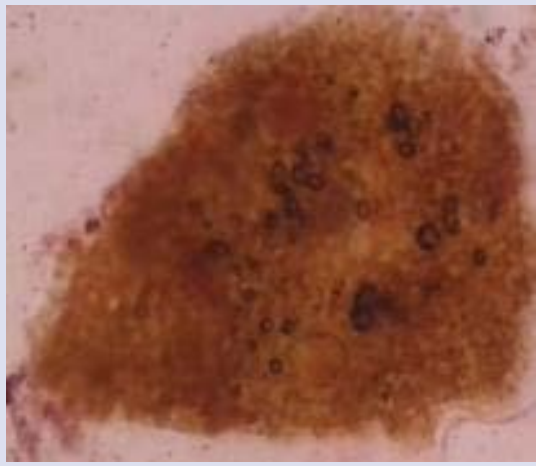

8

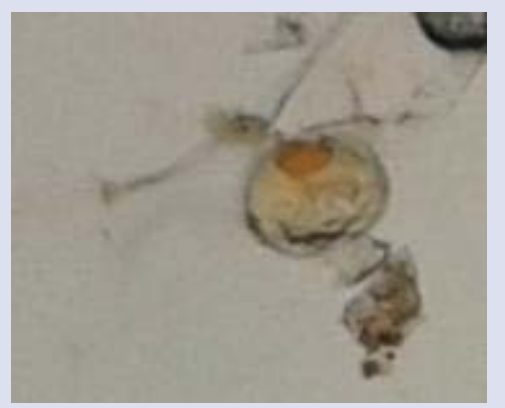

2

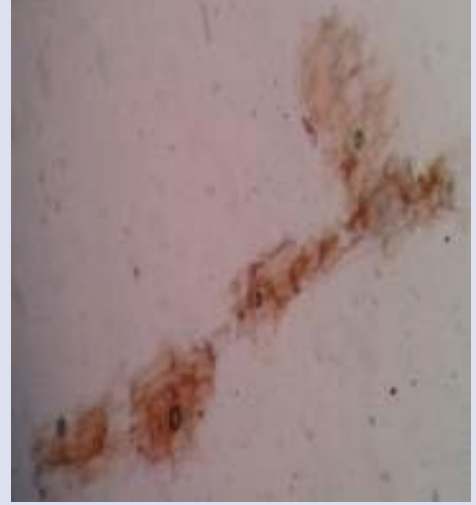

3

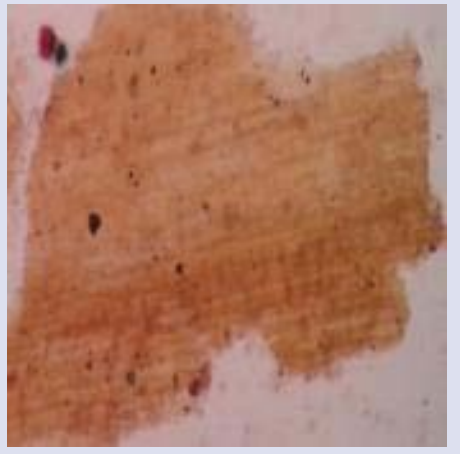

6

Ocimum gratissimum: 1-Reticulate xylem vessel, 2-Fiber, 3- Cortical cell, 4-Stone cell, 5-Multicelluler trichome, 6-Epidermal cell, 7Vessels, 8-Oil glands

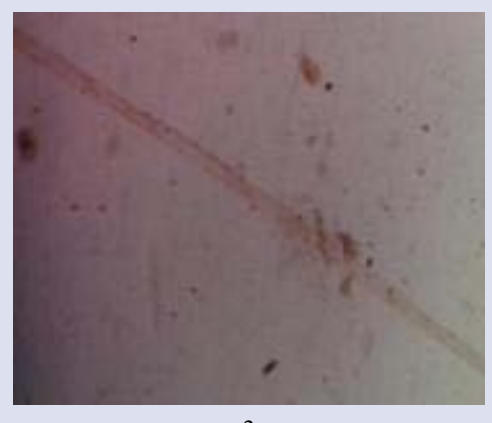

3 


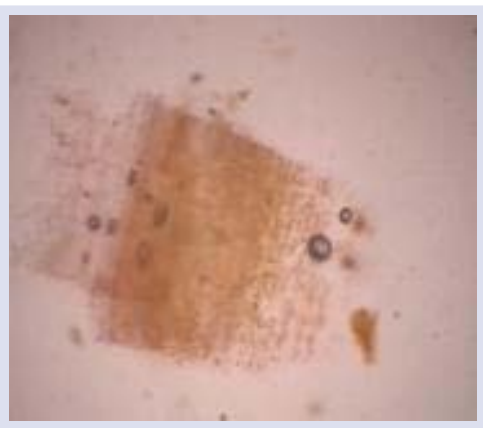

4

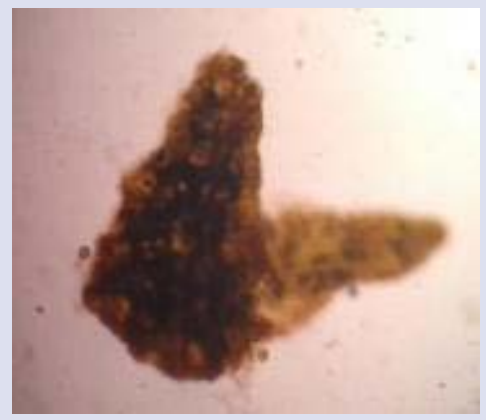

5

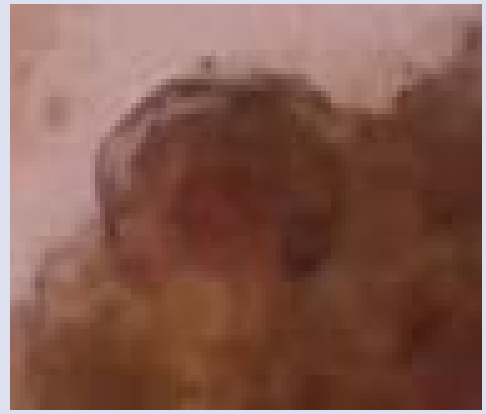

6

Ocimum sanctum : 1- Multicelluler curved trichomes, 2-Glandular trichomes, 3-Scleranmatous fiber, 4-Epidermal cell, 5-Cork cell, 6-Bicuspid stomata
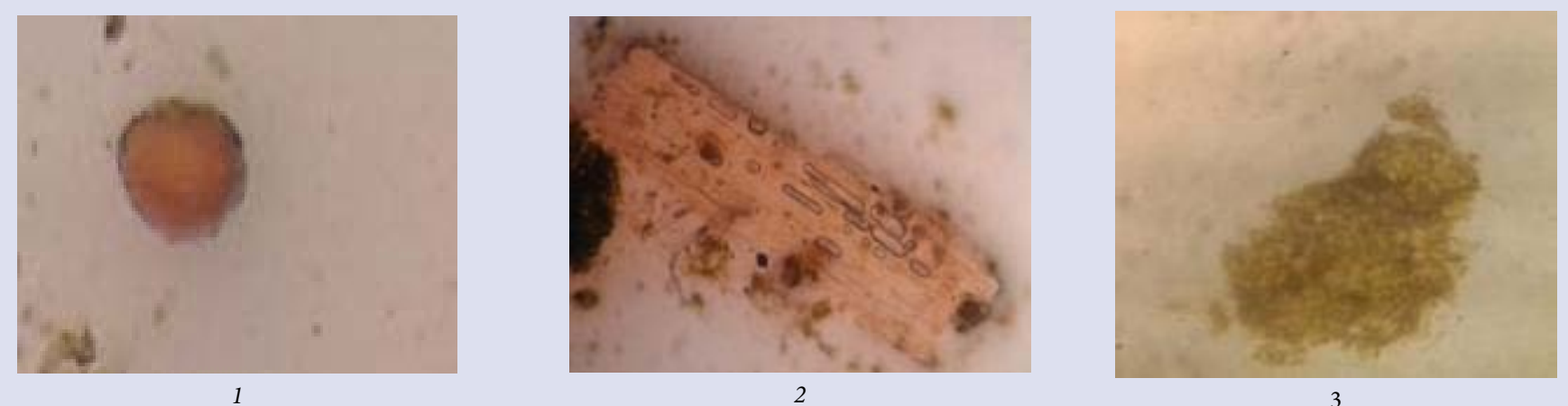

3

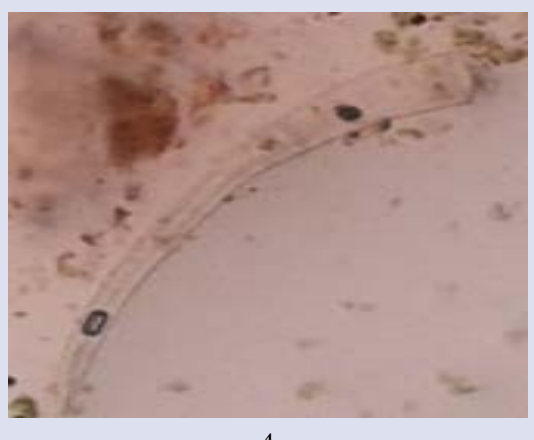

Ocimum kilimandscharicum:1- Stone cell, 2-Reticulate xylem vessel, 3-multi cellular curved trichomes, 4-Storage parenchyma,

end, multicelluler covering trichome, Diacytic stomata, Wavy walled epidermal cells, Collenchymatous cell with intercellular spaces, Vessels. This species can be identified as highly multi-cellular trichome containing species. This is only one species which contain trichomes on the stem.

Ocimum kilimandscharicum leaf part shows multi-cellular covering trichomes, multi-cellular curved trichomes, upper epidermis upper palsied cell, vascular bundle containing xylem \& phloem, Collenchymatous cells, and stem part shows cork, Collenchymatous cells, vascular bundle containing xylem \& phloem, multi-cellular curved trichomes, pith and powder microscopy shows Storage parenchyma, Reticulate xylem vessel, multi cellular curved trichomes, Stone cell.

Ocimum sanctum leaf microscopy shows Unicellular covering trichomes, Glandular trichomes, Upper epidermis, Upper palsied cell, Vascular bundle containing xylem \& phloem, collenchymatous cells, Parenchyma cell, Epidermal cell. Stem shows cork, collenchymatous cells, pith, and medullar rays. Powder microscopy shows, glandular trichomes, fiber multi-cellular curved trichomes, sclerenchymatous fiber, and stomata cell cork cell bicuspid epidermal cells.

Physicochemical evaluation (Tables 4 \& 5) was carried out which shows that total ash values ranges from 7.6 (O. basilicum) to 8.7 (O. sanctum) $\% \mathrm{w} / \mathrm{w}$, acid insoluble ash ranges from 0.2 to $0.4 \% \mathrm{w} / \mathrm{w}$, water soluble ash ranges from 3 to $3.7 \% \mathrm{w} / \mathrm{w}$. Extractive values are found asalcohol soluble extractive value ranges from 2.9 (O. sanctum) to 5.7 (O. kilimandscharicum) $\% \mathrm{w} / \mathrm{w}$, water soluble extractive value which ranges from 3.7 (O. americanum) to 6.9 (O. sanctum) \% w/w and ether soluble extractives which ranges from 2.1 (O. kilimandscharicum) to 3.8 (O. basilicum) $\% \mathrm{w} / \mathrm{w}$.

The phytochemical studies were carried to collect the comparative phytochemical analysis of selected species of Ocimum. Phytochemical tests were carried for presence of alkaloids, glycoside, anthraquinone glycosides, gums mucilage, proteins, amino acids, tannins, phenolic compound, triterpenoids, steroids, sterols, saponins, flavones, flavonoids. Results are summarised in Table 6.

For the extraction of essential oils, shaded dried powder of whole plant was used. Essential oil was extracted by using Clevenger apparatus and oil was stored in air tight amber colored bottle.

Comparative chromatographic evaluation (Table 7 and Figure 1) was carried out for methanolic extract and essential oils of all five species using silica gel $\mathrm{GF}^{254}$ as a stationary phase and toluene: ethyl acetate (93:7) as mobile phase.

In-vitro Antioxidant activity was carried out by DPPH (1, 1- diphenyl-2picryl-hydrazyl) method. Antioxidant activity observed as shown in 
Table 4: Comparative ash values of selected five Ocimum species.

\begin{tabular}{|c|c|c|c|}
\hline Ocimum species & $\begin{array}{c}\text { Total Ash } \\
(\% w / w)\end{array}$ & $\begin{array}{c}\text { Acid Insoluble } \\
(\% \mathrm{w} / \mathrm{w})\end{array}$ & $\begin{array}{c}\text { Water Soluble } \\
(\% \mathrm{w} / \mathrm{w})\end{array}$ \\
\hline Ocimum americanum & 8.1 & 0.4 & 3.8 \\
\hline Ocimum basilicum & 8.7 & 0.2 & 3.5 \\
\hline Ocimum gratissimum & 7.9 & 0.3 & 3.8 \\
\hline Ocimum kilimandscharicum & 7.6 & 0.3 & 3.0 \\
\hline Ocimum sanctum & 8.3 & 0.4 & 3.7 \\
\hline
\end{tabular}

Table 5: Comparative extractive values selected five Ocimum species.

\begin{tabular}{|c|c|c|c|c|c|}
\hline Extractive values & $\begin{array}{l}\text { Ocimuma } \\
\text { mericanum }\end{array}$ & $\begin{array}{l}\text { Ocimum } \\
\text { basilicum }\end{array}$ & $\begin{array}{l}\text { Ocimum } \\
\text { gratissimum }\end{array}$ & $\begin{array}{c}\text { Ocimum } \\
\text { kilimandscharicum }\end{array}$ & Ocimum sanctum \\
\hline Water soluble & 3.7 & 6.9 & 6.4 & 4.0 & 4.5 \\
\hline Alcohol soluble & 2.9 & 4.2 & 3.0 & 5.7 & 2.7 \\
\hline Ether soluble & 3.5 & 3.8 & 3.2 & 2.1 & 3.1 \\
\hline
\end{tabular}

\section{Table 6: Comparative preliminary phytochemical screening results of selected five Ocimum species.}

\begin{tabular}{|c|c|c|c|c|}
\hline Ocimum species & \multicolumn{4}{|l|}{ Phytochemicals } \\
\hline Ocimum americanum & \multicolumn{4}{|c|}{$\begin{array}{l}\text { Alkaloids, Glycoside, Gums mucilage, Proteins, Amino acids, Tannins, Phenolic compounds, Triterpenoids, Volatile oils, } \\
\text { Steroids, Sterols, Saponins, Flavones, Flavonoids }\end{array}$} \\
\hline Ocimum basilicum & \multicolumn{4}{|c|}{$\begin{array}{l}\text { Alkaloids, Glycoside, Gums mucilage, Proteins, Amino acids, Tannins, Triterpenoids, Volatile oils, Steroids, Sterols, } \\
\text { Saponins, Flavones, Flavonoids }\end{array}$} \\
\hline Ocimum gratissimun & \multicolumn{4}{|c|}{$\begin{array}{l}\text { Alkaloids, Proteins, Amino acids, Phenolic compounds, Tannins, Triterpenoids, Volatile oils, Steroids, Sterols, Saponins, } \\
\text { Flavones, Flavonoids }\end{array}$} \\
\hline Ocimum kilimandschari & \multicolumn{4}{|c|}{ Alkaloids, Glycoside, Gums mucilage, Proteins, Amino acids, Triterpenoids, Volatile oils } \\
\hline Ocimum sanctum & \multicolumn{4}{|c|}{$\begin{array}{l}\text { Alkaloids, Glycoside, Gums mucilage, Proteins, Amino acids, Tannins, Phenolic compounds, Triterpenoids, Volatile oils } \\
\text { Steroids, Sterols, Saponins, Flavones, Flavonoids }\end{array}$} \\
\hline \multirow{2}{*}{ Ocimum species } & \multicolumn{2}{|c|}{ Essential Oils } & \multicolumn{2}{|c|}{ Methanolic extract } \\
\hline & Total Separated constituents & Rf Value & Total Separated constituents & Rf Value \\
\hline Ocimum americanum & 07 & $\begin{array}{l}0.23,0.33,0.45,0.51,0.73,0.84 \\
0.98\end{array}$ & 03 & $0.1,0.2,0.3$ \\
\hline Ocimum basilicum & 10 & $\begin{array}{l}0.1,0.18,0.19,0.22,0.33,0.41 \\
\quad 0.48,0.70,0.8,0.9\end{array}$ & 04 & $0.08,0.15,0.17,0.24$ \\
\hline Ocimum gratissimum & 00 & Nil & 02 & $0.15,0.3$ \\
\hline Ocimum kilimandscharicum & 02 & $0.3,0.6$ & 01 & 0.15 \\
\hline Ocimum sanctum & 09 & $\begin{array}{l}0.08,0.1,0.22,0.21,0.3,0.4,0.7 \\
0.8,0.9\end{array}$ & 02 & $0.1,0.25$ \\
\hline
\end{tabular}

Table 8: Comparative Antioxidant potential of selected five Ocimum species.

\begin{tabular}{cccc}
\hline No. & Extracts & $\begin{array}{c}\text { IC } 50 \\
{[\mu \mathrm{g} / \mathrm{ml}]}\end{array}$ & Antioxidant Activity (\%) \\
\hline 1. & Ascorbic acid $(10 \mu \mathrm{g} / \mathrm{ml})$ & 1.83 & 3.1 \\
2 & Ocimum americanum & 3.1 & 36.3 \\
3 & Ocimum basilicum & 1.8 & 36.3 \\
4 & Ocimum gratissimum & 1.8 & 18.1 \\
5 & Ocimum kilimandscharicum & 1.2 & 18.1 \\
6 & Ocimum sanctum & 39.2 \\
\hline
\end{tabular}

Table 9: Comparative antimicrobial potential of selected five Ocimum species.

\begin{tabular}{|c|c|c|c|c|c|c|}
\hline Bacteria & $\begin{array}{c}\text { Ocimum } \\
\text { americanum }\end{array}$ & $\begin{array}{c}\text { Ocimum } \\
\text { basilicum }\end{array}$ & $\begin{array}{c}\text { Ocimum } \\
\text { gratissimum }\end{array}$ & $\begin{array}{c}\text { Ocimum } \\
\text { kilimandscharicum }\end{array}$ & Ocimum sanctum & Standaro \\
\hline Bacteria (E coli) & $20 \mathrm{~mm}$ & $19 \mathrm{~mm}$ & $31 \mathrm{~mm}$ & $10 \mathrm{~mm}$ & $13 \mathrm{~mm}$ & $33 \mathrm{~mm}$ \\
\hline $\begin{array}{c}\text { Fungus (Candida } \\
\text { albicans) }\end{array}$ & - & $31 \mathrm{~mm}$ & - & - & $34 \mathrm{~mm}$ & $32 \mathrm{~mm}$ \\
\hline
\end{tabular}




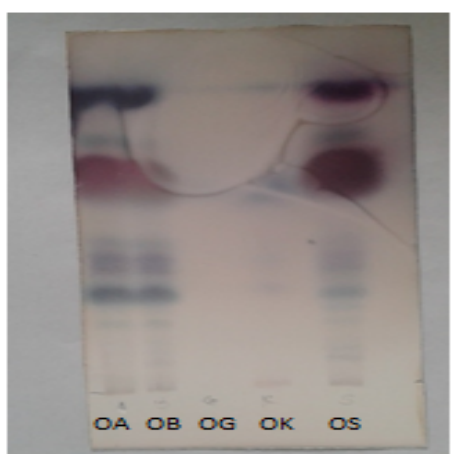

TLC of oils

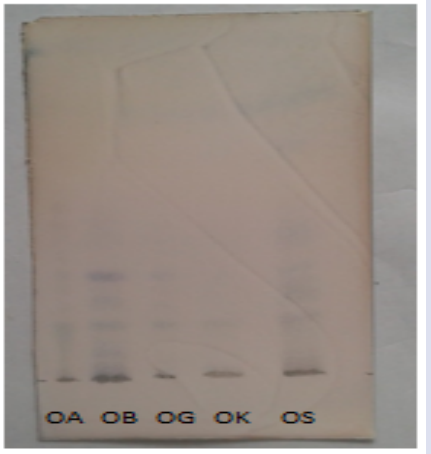

TLC of extracts

Figure 1: TLC of essential oil and extract where $\mathrm{OA}$ : Ocimum americanum, $\mathrm{OB}$ : Ocimum basilicum, OG: Ocimum gratissimum, OK: Ocimum kilimandscharicum, OS: Ocimum sanctum.

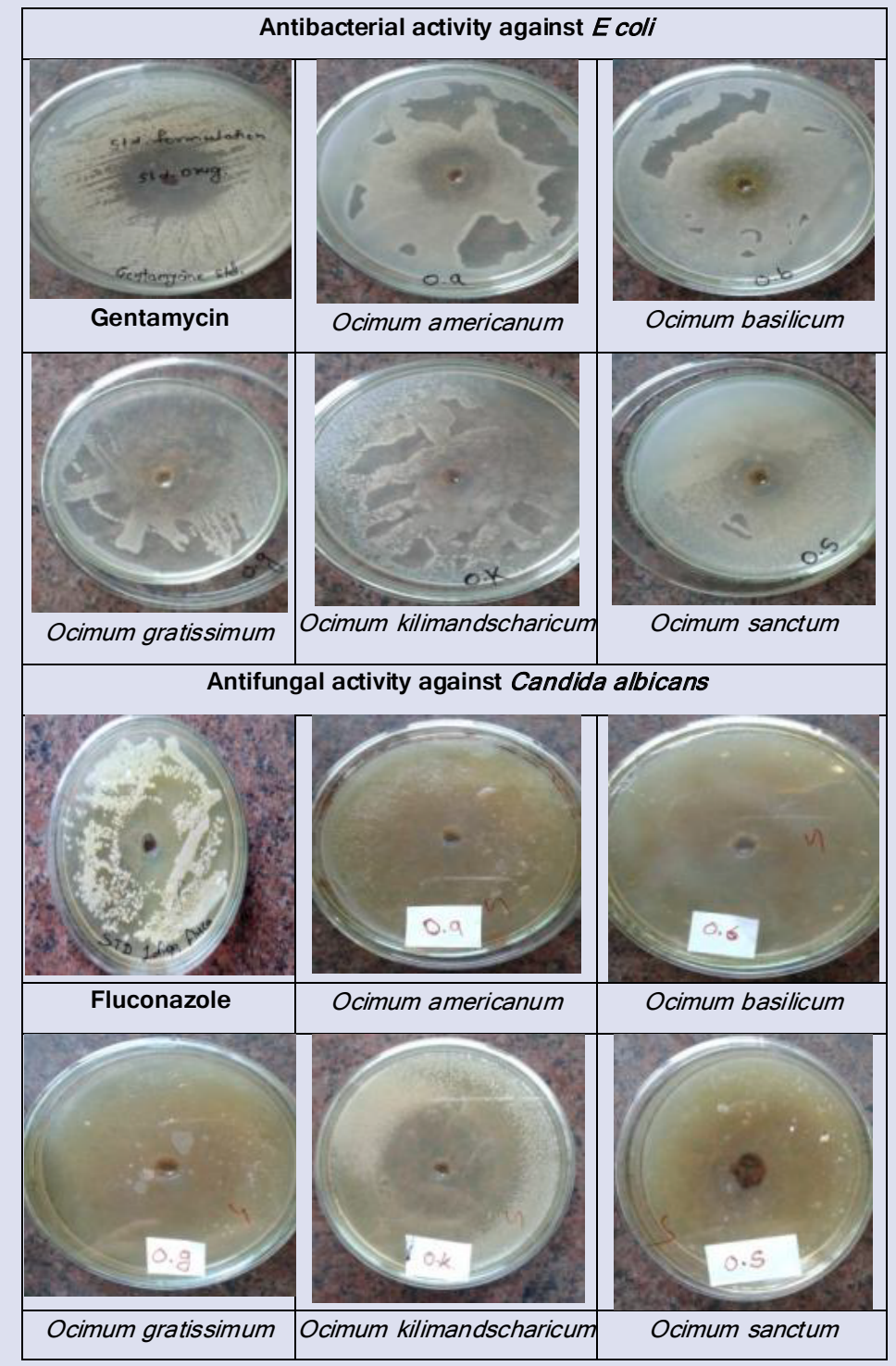

Figure 2: Comparative antimicrobial potential of selected five Ocimum species. 
Table 8 more in O. sanctum while O. basilicum and O. gratissimum also showed good anti-oxidant activity.

Antimicrobial potential (Table 9 and Figure 2) was evaluated by agar well method against $E$. coli and Candida albicans O. kilimandscharicum has more potent antifungal activity compared to O.americanum, $O$. basilicum, O.gratissimum, O.sanctum while later species have more antibacterial activity.

\section{CONCLUSION}

Present work has provided useful information to identity, differentiate and evaluate most commonly used and confusing specis of genus Ocimum-O. americanum, O.basilicum. gratissimum, O. kilimandscharicum, O.sanctum. Finally it can be concluded that $O$. sanctum should be preferred among five selected species based on comparative phytochemical composition, antioxidant and antimicrobial activity.

\section{CONFLICTS OF INTEREST}

There is no conflict of interest.

\section{FINANCIAL SUPPORT AND SPONSORSHIP}

Nil.

\section{REFERENCES}

1. The Wealth of India, a dictionary of Indian raw materials \& industrial products; Raw Materials VII:N-Pe. NISCAIR (CSIR), New Delhi. p.418-419; 1966.

2. Sharma $V_{1}$ Joshi A, Dubey BK. Comparative pharmacognostical and phytochemical evaluation (leaf) of different species of Ocimum. International Journal of Phytopharmacy. 2011;1(2):43-9.

3. Choudhury GB, Behera M, Kumar JP, Tripathy SK. Pharmacognostical and phytochemical investigation of various Tulsi plants available in south eastern Odisha. International Journal of Research in Pharmaceutical and Biomedical Sciences. 2011;2(2):60-3

4. Sahu PK., Kumari A, Sao S, Singh M and Pandey P. Sacred plants and their Ethno-botanical importance in central India: A mini review. International Journal of Pharmacy \& Life Sciences. 2013;4(8):2910-4.

5. Patel DS, Khare PK. Chaurasia B. Identification of morphologically Close Species of Ocimum L. on the basis of seed characters. Indian Journal of Plant Sciences. 2015;4(1):16-8

6. Pandey AK, Singh P, Tripathi NN. Chemistry and bioactivities of essential oils of some Ocimum species: an overview. Asian Pacific Journal of Tropical Biomedicine. 2014:(4):682-94

7. Dolly G, Soni N, Raheja S, Agrawal S. Ocimum kilimandscharicum: a systematic review. Journal of Drug Delivery \& Therapeutics. 2012;2(3):45-52.

8. Rahman S, Islam R, Kamuzzaman M, Alam K and Jamal AHM. Ocimum sanctum $L$ : A review of phytochemical and pharmacological profile. American journal of drug discovery and Development. 2011;4(7):95-105.

9. Joshi RK, Hoti SL. Chemical composition of the essential oil of Ocimum tenuiflorum L. (Krishna Tulsi) from North West Karnataka, India. Plant Science Today. 2014;1(3):99-102.
10. Behera S, Manohar BS, Roja RY, Choudhury PK, Panigrahi R. Phytochemical investigation and study on antioxidant properties of Ocimum canum hydroalcoholic leaf extracts. Journal of Drug Delivery \& Therapeutics. 2012;2(4):122-8.

11. Patil DD, Mhaske DK, Wadhawa GC. Antibacterial and Antioxidant study of Ocimum basilicum Labiatae (sweet basil). Journal of Advanced Pharmacy Education \& Research. 2011;( 2):104-12.

12. Prabhu KS, Lobo R, Shirwaikar AA, Shirwaikar A. Ocimum gratissimum: A Review of its Chemical, Pharmacological and Ethnomedicinal Properties. The Open Complementary Medicine Journal. 2009;(1):1-15.

13. Shukla A, Kaur K, Ahuja P.Tulsi-The medicinal value. International Interdisciplinary Research Journal. 2013;3(2):9-14.

14. Kirtikar KR, Basu BD. Indian Medicinal Plants with Illustrations. 2nd edition. Uttaranchal: Oriental Enterprises. 2003;8:2701-5.

15. The Ayurvedic Pharmacopoeia of India Part - II (Formulations) volume - II First edition, Government of India Ministry of Health and Family Welfare, Department of AYUSH, New Delhi, p.170-176;2008.

16. Khurram SM, Khaliqurrehman A, Khan FA. Comparative Analyses of Ocimum santum Stem and Leaves for Phytochemicals and Inorganic Constituents Middle-East. Journal of Scientific Research. 2013;(2):236-40.

17. Vani S., Cheng SF, Chuah $\mathrm{CH}$. Comparative Study of Volatile Compounds from Genus Ocimum. American Journal of Applied Sciences. 2009;6(3):523-8.

18. Soran, Maria-Loredana \& Codruta, Cobzac \& Varodi, Codruta \& Lung, Ildiko \& Surducan, Emanoil \& Surducan, Vasile. (2009). The extraction and chromatographic determination of the essentials oils from Ocimum basilicum L. by different techniques. Journal of Physics: Conference Series. 182. 012016. 10.1088/1742-6596/182/1/012016.

19. S. Khair-ul-Bariyah, D. Ahmed and M. Ikram. Ocimum basilicum: A Review on Phytochemical and Pharmacological Studies. Pak. J. Chem. 2012;2(2):78-85.

20. Mahapatra SK, Chakraborty SP, and Roy S. Immunomodulatory Role of Ocimum gratissimum and Ascorbic Acid against Nicotine-Induced Murine Peritoneal Mlular Longevity. 2011:1-11.

21. Etinosa O. Igbinosa, Edwina O. Uzunuigbe, Isoken $\mathrm{H}$. Igbinosa, Emmanue E. Odjadjare, Nicholas O. Igiehon, Oke A. Emuedo. In vitro assessment of antioxidant, phytochemical and nutritionalproperties of extracts from the leaves of Ocimum gratissimum (linn). Afr $\mathrm{J}$ Tradit Complement Altern Med. 2013;10(5):292-8.

22. Singh V, Krishan P, Shri R. Ocimum Kilimandscharicum Guerke: Phytochemical and Pharmacological Aspects: A Review. Research and reviews: Journal of pharmacognosy and phytochemistry. 2014;2(3):95-105.

23. FofieN'guessan Bra Yvette, Coulibaly Kiyinlma, Kone-BambaDiénéba Phamacognostic study of Ocimum gratissimum Linn: Pharma food plant. Journal of Pharmacognosy and Phytochemistry. 2014;2(5):74-9.

24. Reshma K, A $\vee R$, Dines $M$ and Vasudevan DM. Radioprotective effects of Ocimum flavonoids on leukocyte oxidants and antioxidants in oral cancer. Indian Journal of Clinical Biochemistry. 2008;23(2):171-5.

25. Khandelwal KR. Edited by Vrunda K Sethi. Practical Pharmacognosy. 20 edition. Nirali Prakashan, Pune. 2010.

26. Khadabadi SS, Deore SL, Baviskar BA. Experimental Phytopharmacognosy Second edition. Nirali Prakashan, Pune. 2012.

27. Indian Pharmacopoeia. Volume-1. The Indian Pharmacopoeia Commission Ghaziabad, p.45-51; 2007.

28. H. Wagner S. Bladt. Plant Drug Analysis. Second edition. Springer, p.352-354; 2009.

29. Colins CH, Lyne PM, Grange JM. Microbiological methods. 7th Ed. Oxford Butterworth - Heineman. p.178; 1995.

30. Bohlin L, Bruhn JG. Bioassay Methods in Natural Product Research and Drug Development. Springer Netherlands, p.69-70; 2013. 


\section{GRAPHICAL ABSTRACT}

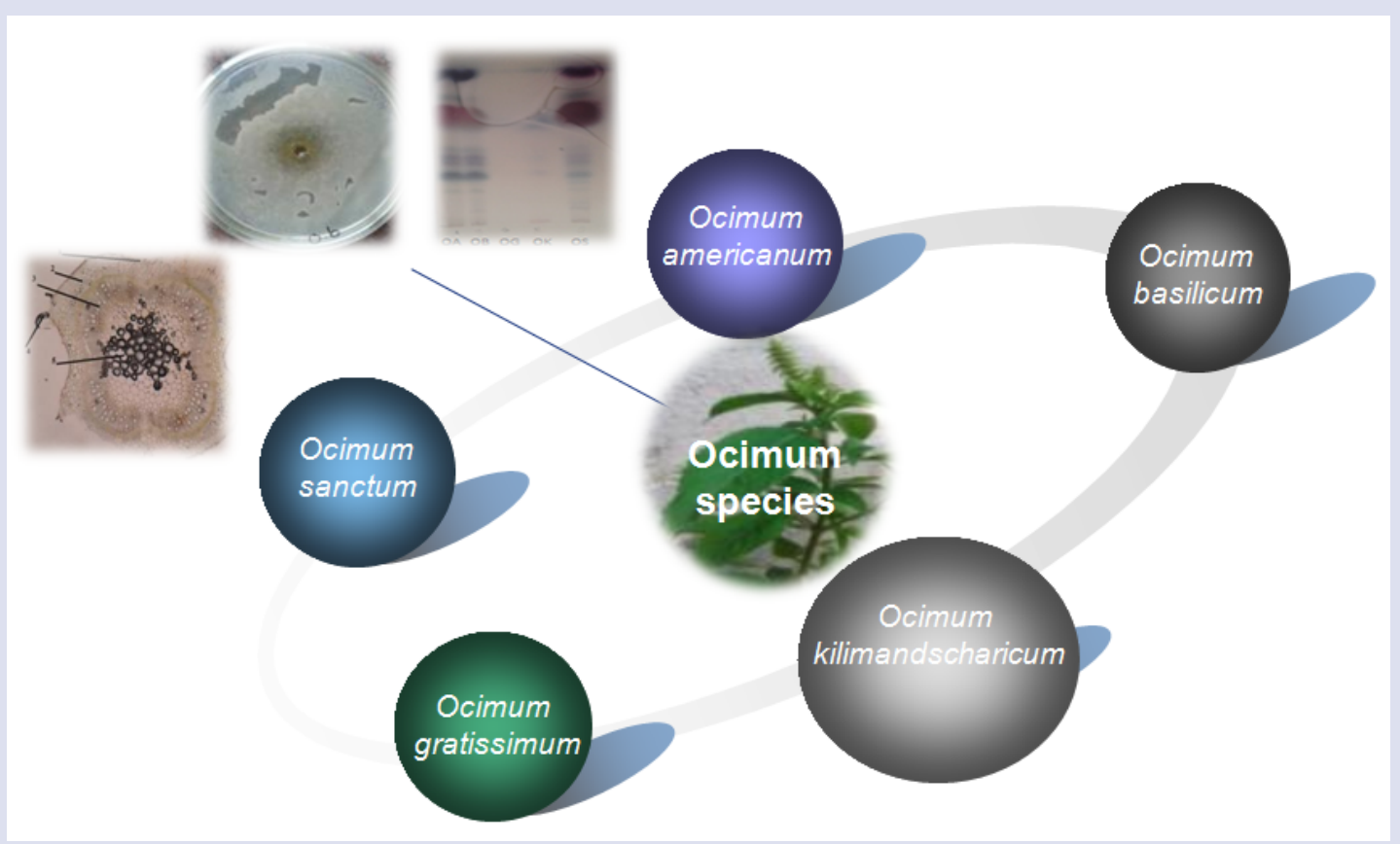

\section{ABOUT AUTHORS}

\section{- Dr. S. L Deore}

Dr. S. L Deore is working with Govt. College of Pharmacy, Amravati (Maharashtra) as Associate professor. She has published many research and review papers in various national- international journals about the natural products. She has authored four books. She has guided many M.Pharm and Ph.D students. She has awarded with Young Scientist Award from AICTE, Delhi. She has fetched about 96 Lakh INR research and medicinal plant campaign grants from various funding agencies like AICTE-Delhi, DST-Delhi, RGSTC-Mumbai and NMPB-AYUSH. She is life member of Society of Pharmacognosy-India, Indian Pharmaceutical Association (IPA) and Association of Pharmaceutical Teachers of India (APTI). Her area of research is isolation and characterization of phytochemicals, nutraceutical development, herbal formulation, chromatographic analysis and screening of extracts.

\section{- Dr. B. A. Baviskar}

Dr. B. A. Baviskar is Assistant Professor at Govt. College of Pharmacy, Amravati (Maharashtra). He has published many research papers in various national- international journals about the medicinal chemistry and natural product chemistry. He has authored three books. He has fetched about 70 Lakh INR research and medicinal plant campaign grants from various funding agencies like DST-Delhi, RGSTC-Mumbai and NMPB-AYUSH. His area of research is synthesis and screening of drugs, computer aided drug design and natural product chemistry.

\section{- Miss Anjali A Kide}

Miss Anjali A Kide has completed M.Pharm in Pharmacognosy and Phytochemistry from Govt. College of Pharmacy, Amravati and now working as JRF in RGSTC, Mumbai funded research project.

\section{- Miss Shital R. Ingole}

Miss Shital R. Ingole has completed M.Pharm in Pharmacognosy and Phytochemistry from Govt. College of Pharmacy, Amravati and currently perusing Ph.D at Govt. College of Pharmacy, Amravati

Cite this article: Deore SL, Ingole SR, Baviskar BA, Kide AA. Comparative Pharmacognostical, Phytochemical and Biological Evaluation of Five Ocimum Species. Pharmacog J. 2021;13(2): 463-74. 